\title{
SYSTEMIC LUPUS ERYTHEMATOSUS MIMICKING HODGKIN'S LYMPHOMA
}

Flavio Barboza ${ }^{1, *}$, Tassia Caroline Beckert Viana ${ }^{1}$, Eduarda Judith Dias Jacome Silva ${ }^{1}$, Raquel Gerep Pereira ${ }^{1}$, Bruna Luiza Oliveira Lima $^{1}$, Thalyne Aparecida Leite de Lima ${ }^{1}$, Maitê Luise Zanette ${ }^{1}$, Vivian de Aquino Medici ${ }^{1}$, Vilian Veloso de Moura Fé ${ }^{1}$, Talles

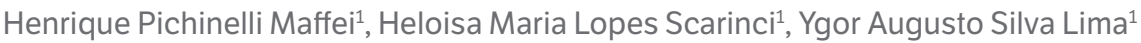

1. Universidade Federal do Mato Grosso, Sinop (MT), Brazil.

*Corresponding author: flavio.fbarboza@uol.com.br

\section{BACKGROUND}

Systemic lupus erythematosus (SLE) is a chronic autoimmune disease with very heterogeneous manifestations. Several conditions promoted by SLE mimic other pathologies, such as hematological diseases. In this context, disorders such as hepatosplenomegaly, present in $27 \%$ of cases, fever in $80 \%$, weight loss and anemia in $60 \%$ of lupus patients, are also present in patients with lymphomas, expanding the range of possible differential diagnoses.

\section{CASE REPORT}

A20-year-old woman starts with an enlarged volume in the left mammary region that, in a breast ultrasound investigation, identified axillary and breast lymph node enlargement. Due to the suspicion of neoplasia, the biopsy performed was compatible with a morphological picture of lymph node showing a predominance of areas with reactive follicular lymphoid hyperplasia and the presence of Hodgkin cells. The subsequent approach was immunohistochemical and PETCT analysis, the latter showing an increase in metabolic activity in lymph nodes in the left pectoral and axillary regions, perigastric and splenic hilum (Fig. 1). While waiting for histochemistry, the patient started with fever and polyarthritis and skin rash. Search for cryoglobulinemias was negative. Immunohistochemistry was negative for lymphomas, malignancy was ruled out, directing the differential investigation to SLE. Additional tests revealed positive homogeneous nuclear FAN 1:640, positive native anti-DNA, positive antinucleosome antibody at high titers, complement consumption (C4) and lymphopenia. In addition, the patient had a good evolution after starting immunosuppressive treatment.

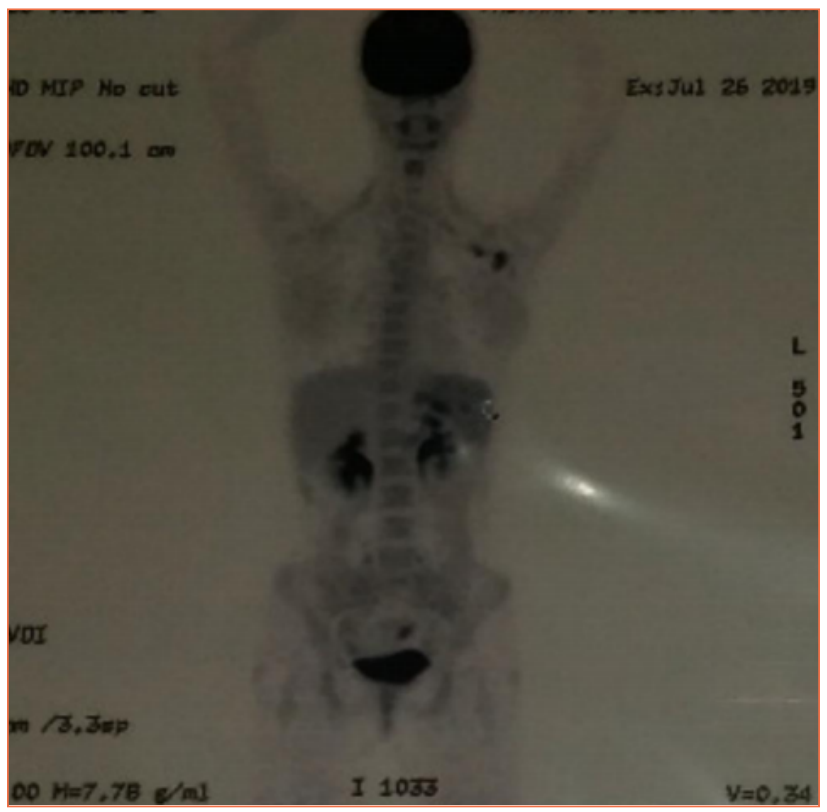

Figure 1. PET-CT showing axillary and around breast lymph node with increased metabolic activity. 


\section{CONCLUSION}

The heterogeneous range of presentation of SLE makes it a diagnosis of exclusion, after investigations that rule out neoplasms and infections. The similarity of hematological and rheumatological disorders (mainly lymph node enlargement) makes immunohistochemistry analysis and quality biopsy essential tools in inconclusive cases. As in this case, in which immunohistochemistry was the differential to close the diagnosis when Hodgkin's lymphoma was removed, suggested by the biopsy report. 